\title{
Les congrès de chimie industrielle et leurs travaux : Une institution de la SCI dans l'entre-deux-guerres
}

\author{
Danielle FAUQUE*
}

\section{Résumé}

La Société de chimie industrielle (SCI), fondée en 1917, a organisé des "congrès de chimie industrielle" annuels qui devinrent rapidement de véritables événements internationaux où des savants, des chercheurs, des politiques rencontraient des industriels et des ingénieurs, non seulement nationaux mais venant aussi d'autres pays industrialisés.

Dans ces congrès, entre les séances plénières et les pauses festives, des questions précises étaient débattues dans les sections thématiques concernées. Pour certains groupes, le congrès était le lieu privilégié où en parler (par exemple : la tannerie, confrontée à l'usage de la nouveauté qu'était le $\mathrm{pH}$ ). D'autres qui rencontraient des difficultés législatives, ou commerciales, établissaient des vœux, envoyés ensuite aux instances de décision (ministères en général).

Comment et sur quels points précis ces groupes travaillaient-ils ? Quels types de vœux formulaient-ils? Quelles relations entretenaient-ils avec d'autres organismes nationaux ou internationaux ?

\section{Mots-clés}

Société de chimie industrielle. Congrès industriels. Union internationale de chimie pure et appliquée (IUPAC). Tannerie. PH. Brevet industriel. Standardisation.

\begin{abstract}
The Société de chimie industrielle (Society for Industrial Chemistry, SCI), founded in 1917, organized annual congresses of industrial chemistry that rapidly became international events. The congresses provided opportunities for members of the scientific and political communities to meet industrialists and engineers, not only from France but also from industrialized nations across the world.
\end{abstract}

\footnotetext{
* Danielle FAUQUE, Chercheur, GHDSO-EST, Université Paris Sud/Paris Saclay. Groupe d'histoire de la chimie - SCF.
} 
Alongside formal plenary sessions and social events, thematic sections allowed for more focused debates. For interest groups, congresses provided a setting in which their own specialities could be addressed: tanning, confronted with recent innovation in techniques for measuring $\mathrm{pH}$, was a case in point. In other sections, legislative or commercial issues could be debated and requests for action drafted, ready for submission to higher authorities, usually government ministries.

How did these groups function, and what subjects did they pursue? What types of request did they formulate? What relationship did they have with other national and international bodies? These are some of the questions that I seek to answer in this paper.

\section{Keywords}

Société de chimie industrielle. Industrial congresses. International Union of Pure and Applied Chemistry (IUPAC). Tannery. PH scale. Patents. Standardisation.

\section{La Société de chimie industrielle, fondatrice des congrès de chimie industrielle}

La Société de chimie industrielle (SCI) est une association 1901, née en 1917 de la volonté de participer à la modernisation de l'industrie chimique française ${ }^{1}$. En 1916, l’État, qui a conscience de la contribution de l'industrie chimique dans tout ce qui est nécessaire à la guerre, va soutenir les organismes les plus à même de travailler à la réorganisation de ce champ à la fois producteur, économique et savant. Étienne Clémentel, arrivé au Ministère du commerce fin 1915, donne l'impulsion nécessaire à la mise en place d'une structure apte à

\footnotetext{
${ }^{1}$ Archives nationales, F/12/7995. Déclaration de l'association : note du 13 mai 1917. Déclaration d'utilité publique le 23 juin 1918. Pour une histoire générale de la SCI, voir Fell Ulrike, "Quelle liaison entre la science et l'industrie ? La Société de chimie industrielle entre les deux guerres, 1917-1939 », in Chimie et industrie en Europe. L'apport des sociétés savantes industrielles du XIX $X^{e}$ siècle à nos jours, Fell U. (dir.), Éditions des archives contemporaines, 2001, p. 69-95 ; Fell U., Disziplin, Profession und Nation. Die Ideologie der Chemie in Frankreich vom Zweiten Paris, Kaiserreich bis in die Zwisschenkriegszeit, Leipziger Universitätsverlag, Leipzig, 2000 .
} 
contribuer au relèvement industriel du pays après la guerre. L'application de méthodes modernes implique la nécessité d'associer recherche scientifique et production, donc d'allier la science et l'industrie, ce que l'Allemagne avait développé depuis des décennies. Les savants et les industriels doivent s'unir pour développer ce programme. C'est ainsi que le Comité consultatif des arts et manufactures, renouvelé, existant depuis 1791, aura en charge d'établir un rapport d'expertise sur les moyens à employer. Ce sera le rapport Clémentel, publié en $1919^{2}$. D'autres instances seront impliquées dans cette entreprise dont la Société d'encouragement pour l'industrie nationale (SEIN) ainsi que la Société des ingénieurs civils. Et c'est dans ce contexte que naît la SCI. Ce projet avait souvent été envisagé avant la guerre. Fondée en 1888, une Société de chimie industrielle de Paris avait eu une brève existence ${ }^{3}$. À la Société chimique de Paris, une section de chimie industrielle avait été créée à l'initiative de Charles Friedel, mais peu fréquentée par les industriels, elle fut supprimée en 1896. Lors des réunions du Syndicat des fabricants des produits chimiques, l'idée avait été régulièrement soulevée, accompagnée d'un projet de création d'une maison de la

\footnotetext{
${ }^{2}$ Letté Michel, « Le rapport d'Étienne Clémentel (1919) : l'avènement administratif des technocrates et de la rationalisation, suivie de l'introduction de ce rapport », Documents pour l'histoire des techniques, 20 ( $2^{\mathrm{e}}$ semestre 2011), CDHSTE-CNAM, SeCDHTE, Paris, 2012, p. 167-195 ; Letté M., « Chimie, chimistes et rationalisation sous les auspices du Ministre du commerce et de l'industrie Étienne Clémentel (1917-1919) », Revue d'histoire des sciences (RHS), 69/1, 19-40 (janvier-juin 2016). É. Clémentel (1864-1936) est un des hommes politiques des plus importants pendant la guerre. De 1915 à 1919, il a été ministre du commerce, de l'industrie et des postes et télégraphes. De décembre 1916 à mars 1917, il est chargé également du portefeuille de l'agriculture et du travail, et de novembre 1917 au 5 mai 1919, du portefeuille des transports maritimes et de la marine marchande.
}

${ }^{3}$ Archives nationales, F12/ 7995. 
chimie. Albin Haller, président du Comité consultatif ${ }^{4}$, avait exhorté les industriels à fonder une telle association, et dès la fin de 1916, l'idée en était fermement acquise ${ }^{5}$. Et cette question avait été discutée lors d'une réunion du Comité le 4 avril $1917^{6}$. Dans cet esprit, la création de la société est décidée le 14 avril 1917, ses statuts déclarés le 23 avril 1917, et placée sous la présidence d'honneur d'Albin Haller et de Henri Le Chatelier et la présidence effective de l'industriel et chimiste Paul Kestner. Jean Gérard, ingénieur-chimiste, en est le secrétaire général. Son siège social est situé 49 rue des Mathurins, dans un quartier d'affaires du VIII ${ }^{\mathrm{e}}$ arrondissement de Paris.

Tirant "les leçons de la guerre", la SCI veut travailler à réformer «notre esprit et notre organisation économique », regrouper les forces, associer les compétences, dans un élan commun et une collaboration étroite de l'industrie et de la chimie. Les moyens pour réaliser ces objectifs prendront plusieurs formes : des congrès, des conférences, des concours, des expositions; un office de chimie industrielle, chargé de fournir des renseignements à la demande et une

\footnotetext{
${ }^{4}$ Albin Haller (1849-1925), professeur de chimie organique à la Faculté des sciences de Paris, directeur de l'ESPCI, membre de l'Institut. Les vice-présidents du Comité sont : Gabriel Chandèze (1846-1926) et Henri Le Chatelier, le rapporteur général Henri Blazeix, les secrétaires Halphen et Léon Bérard. Chandèze est directeur honoraire du Conservatoire des arts et métiers. Bérard, ancien secrétaire du comité, a été nommé par le ministre. Halphen est chef du laboratoire des expertises au Ministère du commerce. Voir Archives nationales, F 12/7995, dossier SCI.

${ }^{5}$ Archives de la Société chimique de France (SCF). Procès-verbaux des séances du conseil, cahier 6. Séance du 8 janvier 1917: "Haller informe le conseil de la fondation prochaine d'une société de chimie industrielle. Il pense que la Société chimique doit rester attentive aux efforts qui sont faits dans cette direction. De même on prête au Syndicat des produits chimiques le projet de créer une sorte de maison du chimiste $»$.
}

${ }^{6}$ Archives nationales, F 12/7995, dossier SCI. 
bibliothèque, sera mis à disposition des adhérents; une revue spécifique à large diffusion, sous une forme attrayante, sera publiée. Cette revue, Chimie \& Industrie, « véritable organe de documentation ou d'information et de diffusion » sera la meilleure façon de réaliser l'union de la science et de l'industrie ${ }^{7}$. Publiée à partir de juin 1918, très rapidement elle présente une quantité de pages de publicité qui lui permettront d'assurer financièrement son avenir.

Pour augmenter son influence et développer son programme, la SCI va organiser des congrès et des expositions à partir de 1921 (voir tableau). Les congrès annuels devaient se dérouler alternativement à Paris et dans une grande ville française. Mais à partir de 1926, ils ont aussi lieu dans un autre pays européen. Le choix des villes n'est pas quelconque, d'abord francophones (Bruxelles, 1926 et 1935, Liège 1930, et en partie au Luxembourg, 1928), puis francophiles comme Barcelone (1929), ou Prague (1932) où existe une section tchèque de la SCI, mais surtout ce sont des lieux où l'industrie de la chimie est particulièrement développée. Il était prévu un congrès en Espagne en 1936, un autre à Varsovie en 1939, mais ils furent annulés, l'un à cause de la guerre civile, l'autre à cause de l'entrée en guerre de la France. D'autres congrès se tiennent à l'occasion d'événements organisés par la SCI, ou qu'elle accompagne (voir tableau) : expositions universelles ou internationales; le centenaire de Louis Pasteur (Paris 1923), de Marcelin Berthelot (Paris 1927), des découvertes de Michel-Eugène Chevreul (Paris 1925); en 1928, à Strasbourg, on profite du CCI pour rendre hommage à Paul

\footnotetext{
${ }^{7}$ Chimie \& Industrie (Ch\&I), présentation, plaquette de présentation, décembre 1917. Cette plaquette a été ajoutée au premier numéro de la revue paru en juin 1918.
} 
Schützenberger dont le centenaire sera célébré à Paris en 1929... Ces manifestations ont aussi lieu au moment d'un événement national ou international : expositions coloniales (Marseille, 1922 ; Paris, 1931), Exposition des arts décoratifs et industriels (Paris, 1925), du Centenaire de l'indépendance (Liège, 1930), Exposition universelle et internationale (Bruxelles, 1935), Exposition internationale des arts et des techniques appliqués à la vie moderne (Paris, 1937), etc.

À partir de 1923, les congrès donnent lieu à d'imposants compte rendus, témoignant de l'importance en nombre, en espace, et en échos recueillis, de ces manifestations. Là encore, la SCI témoigne de sa capacité à rassembler dans un même lieu des acteurs d'origine différente: hommes politiques, professeurs de faculté, membres de l'institut, savants renommés, nationaux ou étrangers, qui côtoient les ingénieurs et industriels locaux recevant et faisant visiter leurs usines, prenant en charge les parties festives de l'événement. Les travaux pouvaient se dérouler dans les bâtiments de l'université, d'un institut technique, ou d'une école d'ingénieur. À Paris, le CNAM est un des premiers lieux investis par la SCI. Des médailles d'or honorent également les savants, industriels ou ingénieurs ayant contribué à "l'union de la science et de l'industrie", par exemple : Paul Sabatier (1922), Henri Le Chatelier (1925), Enrique Hauser (1929), Victor Grignard (1931), etc.

Aux congrès, toutes les branches de l'industrie chimique et activités connexes sont représentées selon une classification proche de celle définie par la classification décimale universelle (CDU) proposée par l'Institut international de bibliographie (IIB) de Paul Otlet à Bruxelles. La revue Chimie \& industrie, « organe mensuel de 
la $\mathrm{SCI} »(\mathrm{sic})$, suit ce système décliné très en détail pour les spécialités. La chimie en est sans doute un des domaines où son application est la plus symptomatique, ou la plus représentative. Dès la création de l'office de documentation qui alimente aussi la revue, le classement des monographies, des périodiques, et de chacun de leurs articles, ainsi que chaque dossier documentaire, se fait selon les règles de la CDU, sous un numéro, parfois plusieurs lorsque l'article portait sur plusieurs domaines, avec des aménagements selon l'hyper spécialisation du domaine concerné. Ces cotes sont indiquées dans la revue.

$\mathrm{Au}$ sein de ses réunions, en dehors des temps festifs, les participants exposent et débattent de leurs problèmes communs. Les questions de standardisation, essentielles pour l'ensemble des branches représentées, et le choix des méthodes d'analyse donnent lieu à des discussions réitérées d'année en année. Les nouveautés sont aussi présentées et discutées. Des vœux sont émis, que la SCI propose de soumettre aux instances administratives ou politiques concernées. Chaque congrès est aussi l'occasion de présenter une exposition, le plus souvent spécialisée principalement sur l'outillage de la chimie par exemple la verrerie graduée et normalisée.

Ces congrès prirent rapidement une dimension internationale, et suppléaient dans une certaine mesure à l'absence de congrès international de chimie pure et appliquée, dont le dernier avait été organisé en 1912 à Washington ${ }^{8}$. Après la Grande Guerre, à l'étranger,

\footnotetext{
${ }^{8}$ Thorburn Burns Duncan \& Deelstra Hendrik, " The origins and impact of the international congresses of applied chemistry, 1894-1912», Microchim Acta, 172, 277-283 (2011). Thorburn Burns D. \& Deelstra H., « Establishing a vital tradition :
} 
des congrès de chimie industrielle sont organisés d'une part par la SCI britannique, et d'autre part par la société italienne, congrès auxquels les membres délégués de la SCI française assistaient. À partir de 1920, en Allemagne, la Dechema (Deutsche Gesellschaft für Chemisches Apparatewesen) a organisé des expositions triennales d'appareillage chimique, les Achema, nées d'un besoin de coopération entre l'ingénieur constructeur d'instruments et le chimiste ${ }^{9}$. Il faudra attendre 1936 et le IX ${ }^{\mathrm{e}}$ congrès international de chimie, à Madrid, sous l'égide de l'Union internationale de chimie pour que le cycle des congrès internationaux de chimie reprenne son cours.

Jean Gérard (1890-1956), est, selon ses contemporains, la « cheville ouvrière » des congrès de la SCI. À partir de 1928, leur développement est tel qu'une commission permanente, présidée par Gérard, sera créée pour l'organisation des congrès de chimie industrielle. En 1926, il publie un ouvrage en deux volumes, 19141924. Dix ans d'efforts scientifiques, industriels et coloniaux, pour lequel l'Académie des sciences lui décerne le prix Montyon d'un montant de 1000 f, le 12 décembre $1927^{10}$. Sur plus de 3000 pages, ce monumental travail présente une documentation dont maints aspects ont été abordés dans les congrès. Dans la notice relative à la SCI

the series of international congresses of applied chemistry, 1894-1912 », Chemistry International, 33/4, 11-14 (2011).

${ }^{9}$ Penau H., « La VII ${ }^{\mathrm{e}}$ Achema », Ch\&I, 31/6, 1482-1488 (juin 1934). Achema : Ausstellung für chemisches Apparatewesen. L'article présente un bref historique des Achema.

${ }^{10}$ Jaisson Marie, Fondations, prix et subventions de l'Académie des sciences (19161996), Brepols Publishers, Turnhout, Belgique, 2003, t. 2, p. 802 (Collection Diversis Artibus, 66-1 et 66-2). 
rédigée en 1926, Jean Gérard écrit à propos des congrès de chimie industrielle :

... ces manifestations ont obtenu un succès dépassant les prévisions les plus optimistes. Les adhérents toujours plus nombreux, l'importance sans cesse croissante des délégations étrangères, la valeur des travaux accomplis ont donné à ces Congrès l'allure de véritables réunions internationales et en ont fait les dignes successeurs des Congrès de chimie pure et appliquée dont la série a été close par la guerre.

Chaque année, le Congrès étudie dans ses sections une série de questions d'actualité et les rapports préparés par des spécialistes qualifiés. Les discussions qui les suivent permettent à chacun d'en tirer des conclusions fructueuses. On peut dire que les Congrès de chimie industrielle, par les liens qu'ils créent et les relations qu'ils font naître entre techniciens et industriels, contribuent de la meilleure manière à l'avancement de la science et au progrès de l'industrie ${ }^{11}$.

La matière est si riche qu'il n'est pas possible en un seul article de donner une analyse complète de ces manifestations organisées par la $\mathrm{SCI}^{12}$. Nous allons donc ici nous intéresser à un choix de sujets débattus de manière récurrente dans les groupes techniques réunis.

\footnotetext{
${ }^{11}$ Gérard Jean, «La Société de chimie industrielle », in 1914-1924. Dix ans d'efforts scientifiques, industriels et coloniaux, Gérard J. dir., Chimie et Industrie, Paris, 1926, t. 2, p. 943-948, voir p. 946-947. Le mot technicien n'est pas ici à prendre dans le sens d'aujourd'hui. Dans Chimie \& Industrie, il est souvent utilisé pour désigner celui qui met en œuvre le savoir savant dans le monde industriel, donc recouvre aussi bien la fonction d'ingénieur, du chimiste industriel ou du contremaître.

${ }^{12}$ Voir certains des aspects de ces congrès dans Fauque Danielle, « I - Les congrès de chimie industrielle dans l'entre-deux-guerres: vitrine des relations entre l'industrie, la science et la politique », L'Actualité chimique, 380, 39-44 (décembre
} 


\section{Le premier congrès (1921) : un succès au delà des espérances}

Le premier congrès de chimie industrielle $(\mathrm{CCI})$, organisé par la SCI, se déroule du 9 au 12 octobre 1921 à Paris. Annoncé comme le premier "meeting annuel" de la SCI, il constituera un "véritable congrès de chimie industrielle", présentant 34 sections techniques, où des "questions importantes seront traitées"13. Comme tous les congrès et conférences de l'époque, l'événement commence par une réception le 9 octobre. Le ministre du commerce, Lucien Dior, ouvre le congrès le lendemain, dans le grand amphithéâtre du $\mathrm{CNAM}^{14}$. Paul Kestner accueille ensuite les congressistes, présente le programme, puis insiste sur le rôle moral de la SCI, et sur le rôle de ce congrès, qui, par ses travaux, veut témoigner de l'alliance de la chimie pure et de la chimie appliquée $^{15}$. La séance de clôture du 11 octobre est présidée par Louis Loucheur, ministre des Régions libérées. Un banquet général de deux cents couverts suit, servi au Palais d'Orsay, sous la présidence du ministre de l'agriculture, Edmond Lefebvre du Prey. Le 12 octobre est consacré aux excursions et aux visites d'usine, et le 13 octobre, un groupe de congressistes se rend à Chauny pour visiter le site reconstruit de Saint-Gobain. Deux ans plus tôt, lors de la réunion préparatoire à la fondation de l'Union internationale de chimie pure et

\footnotetext{
2013), (Dossier : Les chimistes, leurs institutions et leurs sociétés savantes entre les deux guerres).

${ }^{13}$ Rubrique information, Chimie \& Industrie (Ch\&I), 6/2, 251 (août 1921).

${ }^{14}$ Lucien Dior (1867-1932), industriel et homme politique. L'usine familiale, Société anonyme des usines Dior, située près de Granville (Manche), est spécialisée dans les superphosphates. Il sera président de la SCI de 1924 à 1927.

${ }^{15}$ Deschiens Maurice, «Premier congrès de la chimie industrielle », Bulletin de la SCI (Bull. SCI), 2, 2-30 (novembre-décembre 1921), voir p. 2-4.
} 
appliquée (UICPA aujourd'hui IUPAC), à Paris, les participants avaient visité les ruines de Chauny ${ }^{16}$.

Une exposition de chimie, sous le patronage du ministère du Commerce s'est tenue au CNAM du 7 au 16 octobre. Représentant deux sections du congrès, la section des appareils de contrôle et d'outillage de laboratoire d'une part, et les matières colorantes d'autre part, elle constitue l'embryon d'une exposition plus complète sur la chimie dans son étendue, prévue dans l'avenir, selon les organisateurs ${ }^{17}$. Une conférence générale a accueilli 3000 personnes dans le grand amphithéâtre de la Sorbonne, la plus grande salle existant à Paris, pour écouter George Claude sur la synthèse de l'ammoniaque $^{18}$. Le succès a été tel, que l'ingénieur a accepté de présenter une deuxième fois sa conférence dans le même amphithéâtre le 14 octobre pour ceux qui n'avaient pu y assister.

Ce premier congrès ne donne pas lieu à un numéro spécial, mais Maurice Deschiens publie un long compte rendu dans le Bulletin de la $S C I^{19}$. Deschiens, ingénieur chimiste, nommé rapporteur général des congrès, fera la totalité des comptes rendus de 1921 à 1938. Victor Cambon, auteur bien connu pour ses études sur l'Allemagne et les

\footnotetext{
${ }^{16}$ Deschiens M., «Visite aux usines de produits chimiques de la Compagnie de Saint-Gobain à Chauny (Aisne) », in " Premier congrès de la chimie industrielle », Bull. SCI, 2, 27-30 (novembre-décembre 1921). Voir aussi «Visite à Chauny » in « Conférence interalliée de chimie, 14-16 avril 1919 », Ch\&I, 2/4, 577-588 (avril 1919).

${ }^{17}$ Voir le compte rendu en partie écrit par André Wahl, professeur de chimie tinctoriale au CNAM, «L'exposition de la chimie », Ch\&I, 6/5, 675-698 (novembre 1921).

${ }^{18}$ Voir à ce sujet : Emptoz Gérard, « II - La nouvelle chimie de l'azote dans l'entredeux-guerres », L'Actualité chimique, 381, 40-46 (janvier 2014), (Dossier: Les chimistes, leurs institutions et leurs sociétés savantes entre les deux guerres).

${ }^{19}$ « Premier congrès », op. cit., note 15, p. 2-30.
} 
États-Unis, rend compte de ce premier congrès en deux pages dans Chimie \& Industrie ${ }^{20}$. Qui, écrit-il, aurait pu prévoir il y a dix ans qu'une association puisse organiser à la fois un congrès, une exposition et une série de représentations cinématographiques représentant des opérations industrielles? Or cela s'est fait. Dans les numéros suivants paraissent les textes des conférences d'intérêt général qui ont été données lors de cette première manifestation ${ }^{21}$. Dans l'intervalle, les groupes de travail se sont réunis par corps de spécialités techniques.

Le style est lancé, définitif. Le succès ira en croissant au long des années qui suivront. Comme chaque congrès s'inscrit dans un événement plus large, il draine de ce fait un public qui dépasse le seul milieu professionnel. Une rencontre avec la presse est aussi systématique. Tous les moyens de communication moderne sont mobilisés pour la conquête de l'opinion publique. Les ministres appuient l'initiative, les délégations étrangères sont nombreuses. Les discours et les toasts invoquant l'alliance de la science et de l'industrie pour le progrès de l'humanité, et la résolution de ses problèmes, sont récurrents; ils prônent aussi le développement d'une organisation scientifique du laboratoire, de l'usine, de la production et de la

\footnotetext{
${ }^{20}$ Cambon Victor, « Les leçons du congrès de chimie industrielle », Ch\&I, 6/4, 417418 (octobre 1921).

${ }^{21}$ Notamment les conférences de William Pope, " L'avenir de la chimie organique », Ch\&I, 6/4, 419-422 (oct. 1921) ; de Henri Le Chatelier , « La méthode scientifique et son application à la chimie analytique », op. cit., p. 423-429; et de Henry Gall (1862-1930), ancien président de la Société des ingénieurs civils, "L'industrie française de la cynamide », op. cit., p. 430-439. Camille Matignon (1867-1934) parle de « l'État et le développement de l'industrie de l'azote », non publié.
} 
documentation. Et ils soulignent, bien évidemment le rôle de la SCI comme acteur incontournable dans la réalisation de ces objectifs ${ }^{22}$.

Les thèmes sont répartis en quinze groupes (voir tableau 2). Certains vont exprimer des vœux, que la SCI relayera vers les instances compétentes. Le mot «standard» est un mot-clé du compte rendu du travail des groupes. Le premier groupe, Chimie analytique, reprend les demandes déjà formulées au cours des deux conférences internationales de chimie (CIC) de 1920 et $1921^{23}$, portant sur la préparation d'échantillons standards de référence, la définition des réactifs pour analyse, l'unification des méthodes d'analyse. En particulier, l'étude de la normalisation de l'analyse physicochimique va être un point fort des activités des CCI, en lien avec l'Union. Cependant, le groupe des chimistes français a trouvé au CCI un cadre plus propice à ses discussions, moins soumis à des contraintes administratives ou diplomatiques qu'au sein de l'Union internationale. C'est clairement sous-entendu dans l'article publié à la suite des deux premières CIC, dans Chimie \& Industrie ${ }^{24}$, et selon les auteurs, André

\footnotetext{
${ }^{22}$ Fauque D., « Vitrine », op. cit. note 12.

${ }^{23}$ Les conférences internationales de chimie sont une émanation de l'Union internationale de chimie pure et appliquée (UICPA). Chaque conférence se déroule dans un pays différent, chaque année de 1920 à 1928, puis tous les deux ans. À cette occasion, les commissions et le conseil de l'Union tiennent leurs réunions et rendent compte au cours des assemblées générales. Voir Fauque D., «Jean Gérard, Secretary-General and Driving Force of the International Chemical Conferences between the Wars ", in Transformation of Chemistry from the 1920s to the 1960s, Kaji Masanori, Furukawa Yasu, Tanaka Hiroaki, Kikuchi Yoshiyuki (editors), Japanese Society for the History of Chemistry, Tokyo, 2016, p. 42-49 (Proceedings of the International Workshop on the History of Chemistry, IWHC 2015 Tokyo 2015). Open access : http://kagakushi.org/iwhc2015/proceedings

${ }^{24}$ Kling André et Lassieur A., « Revue analytique », Ch\&I, 6/6, 746-764 (décembre 1921).
} 
Kling et A. Lassieur, les chimistes analystes ont pu, dans le cadre du CCI, sortir de leur isolement et régler leurs affaires par eux-mêmes.

Le groupe III, sur les combustibles, émet le vœu que lors du prochain congrès international du pétrole, soit créé un Institut international du pétrole dont le siège serait à Strasbourg ${ }^{25}$. Le groupe XII, sur la tannerie, propose une nouvelle méthode, préconisée par la commission d'analyse du cuir de la Section française de l'Association internationale des industries du cuir. Cette nouvelle méthode fait appel à la concentration en ions hydrogène et le dosage de cette concentration par le $\mathrm{pH}$ : «c'est le $\mathrm{pH}$ que le tanneur » doit connaître en place de l'acidité du jus de tannerie. « Le rôle du pH commence à jouer un rôle capital dans la tannerie ». On souhaite : « que le prochain congrès de la SCI coïncide avec une réunion plénière de la Section française de la Société [internationale] des chimistes de l'industrie du cuir », dont plusieurs de ses membres appartiennent d'ailleurs à la SCI, dont J. Gérard, fils d'Edmond, tanneur à $\mathrm{Nancy}^{26}$. Gérard avait d'ailleurs contribué à l'organisation dès 1919 de la première réunion interalliée de l'Association internationale du cuir, refondée après la guerre, excluant les Allemands ${ }^{27}$. Effectivement, cette section française tiendra ensuite ses assemblées plénières lors des CCI.

Des vœux de collaboration sur des points de chimie appliquée sont émis à l'intention de l'Union internationale de chimie, notamment l'établissement de normes internationales en matière de

\footnotetext{
25 « Premier congrès », op. cit. note 15, p. 9. Un Institut français du pétrole avait été créé en 1919, puis fut transféré à Strasbourg en 1922.

${ }^{26}$ Ibid., p. 11.

${ }^{27}$ Gérard J., «Le congrès de la Société des chimistes du cuir, 22-23 septembre $1919 »$, Ch.\&I., 2/9 (septembre 1919), voir p. I-IV intra p. 1004-1005.
} 
conservation des matières alimentaires par des procédés tant physiques que chimiques. La technique du froid donnera lieu à des réunions spécifiques dans les futurs CCI.

D'autres vœux sont émis, concernant l'industrie des matières colorantes en France qui souffre à nouveau d'une forte concurrence allemande $^{28}$ et la formation des chimistes, nécessitant une réforme de l'enseignement, des brevets, et une protection équitable permettant la survie et le développement de l'industrie française ${ }^{29}$.

Enfin, la question de la documentation chimique est clairement posée, et la création d'une Maison de la Chimie est vivement souhaitée par tous. Elle abriterait les services de l'Office de documentation scientifique, technique et économique, organisme créé par la SCI et «qualifié par le nombre important de journaux qu'il reçoit pour servir de base au système de la documentation chimique en langue française ». On formule à cet effet le vœu de s'entendre avec la $\mathrm{SCF}$, afin d'éviter les doublons et les frais d'extraction selon la nature

\footnotetext{
${ }^{28}$ Voir aussi Frossard Joseph, « La protection de l'industrie des matières colorantes en France », $C h \& I, 6 / 4,533-535$ (octobre 1921). Cet article fut suivi de celui de E.V. Evans, "La protection des matières colorantes en Grande-Bretagne », Ch\&I, 5/5, 669-674 (novembre 1921). La Society of Chemical Industry (SCIb), société sœur, entretenait des liens étroits avec la SCI française depuis sa création grâce aux liens personnels de Paul Kestner avec Henry Louis, son président. À chaque réunion de l'une ou de l'autre des deux sociétés, des délégués étaient présents. L'industrie des matières colorantes représente un problème récurrent pour la France, voir Béhal Auguste, Moureu Charles et Haller Albin, «La crise de notre industrie chimique organique et la Défense nationale », Revue scientifique, 60/20, 681-684 (28 octobre 1922). Voir Langlinay Erik, "Kuhlmann at War (1914-1924)», in Frontline and Factory. Comparative Perspectives on the Chemical Industry at War, 1914-1924, MacLeod Roy and Johnson Jeffrey A., eds, Springer, Dordrecht, 2006, p. 145-166.

${ }^{29}$ « Premier congrès », op. cit., note 15, p. 11-12.
} 
« science pure » ou «science appliquée ${ }^{30}$. Ce dernier vœu restera lettre morte.

\section{Le deuxième Congrès et l'Union internationale de chimie (1922)}

Le deuxième CCI se tient à Marseille du 2 au 8 juillet 1922. Il mérite une étude particulière compte tenu des circonstances dans lesquelles il s'est déroulé, car il suit exactement la troisième Conférence internationale de chimie (CIC) à Lyon, du 27 juin au $1^{\text {er }}$ juillet. À la fin de cette $\mathrm{CIC}$, les congressistes sont invités à descendre le Rhône en bateau jusqu'à Avignon, puis prennent le train et le bus pour atteindre Marseille, voyage organisé par le Comité lyonnais d'organisation de la CIC. Cette présence de savants et industriels étrangers est hautement soulignée dans l'introduction au compte rendu qui paraît dans Chimie \& Industrie du mois d'août ${ }^{31}$. Comme l'an passé, des ministres y sont invités.

Le 3 juillet, la séance d'ouverture se déroule dans l'amphithéâtre des sciences naturelles de la nouvelle faculté des sciences, dont le doyen Paul Rivals est fier de montrer la modernité. La Chambre de commerce de Marseille avait décidé en 1917 la

\footnotetext{
${ }^{30}$ Ibid., p. 12. À propos de l'office de documentation chimique, voir Fauque D., « La documentation au cœur de la réorganisation de la chimie dans l'entre-deuxguerres: Rôle des sociétés savantes et institutions françaises dans le contexte international », RHS, 69/1, 41-75 (janvier-juin 2016). Pour la Maison de la Chimie, voir Fox Robert, « Science, celebrity, diplomacy : the Marcellin Berthelot centenary, 1927 », RHS, 69/1, 7-115 (janvier-juin 2016) ; voir aussi Fauque D., « Introduction : Aux origines de la Maison de la Chimie », RHS, 69/1, 5-17 (janvier-juin 2016).

${ }^{31}$ En particulier, parmi les étrangers, signalons la présence d'Emmanuele Paternò, sénateur, président du Conseil italien de chimie, William A. Noyes, ancien président de l'American Chemical Society, des délégués des divers pays d'Europe, du Japon, du Canada, d'Argentine. Voir Deschiens M., «Deuxième Congrès de la chimie industrielle », $C h \& I, 8 / 2,217-268$ (août 1922), voir p. 219.
} 
création d'un Institut technique supérieur, dont Rivals est le directeur, comportant une école de chimie, un cours d'enseignement professionnel, une section économique, et un laboratoire d'essais techniques. C'est dans cet institut que les groupes techniques du congrès ont travaillé.

Professeur de chimie industrielle, Rivals avait contribué au déroulement de plusieurs manifestations savantes pour faire connaître cet établissement dans le but d'obtenir des moyens plus importants de développement. L'influence de la conférence de Lyon s'est particulièrement fait sentir dans les travaux du groupe XIV sur la question de la conservation des matières alimentaires par les procédés physiques et chimiques, question déjà largement débattue quelques jours plus tôt à Lyon ${ }^{32}$. Au CCI, le groupe IX sur les matières grasses a été également particulièrement actif.

Lors de la séance de clôture, le président de la Chambre de commerce de Marseille, Hubert Giraud, présentait ce qui différenciait Lyon de Marseille, la première pour les produits de luxe et produits finis dont le chiffre d'affaires atteignait plusieurs millions de francs, la seconde pour les produits de base et d'importantes transformations de matières premières mettant en jeu des millions de tonnes, illustrant alors les rôles respectifs du praticien et du savant. La conférence de Paul Sabatier, sur l'hydrogénation catalytique des corps gras, a encore accentué le rôle du savant dans l'évolution des méthodes industrielles. Le ministre des colonies, Albert Sarraut, ancien ministre de

\footnotetext{
${ }^{32}$ Paternò Emmanuele, « Conservation des matières alimentaires par des substances chimiques », in Compte rendu de la troisième conférence internationale de la chimie, Paris, Secrétariat général Jean Gérard, 1923, p. 56, et p. 123-124. Dans la suite des notes, cette publication régulière sera notée : CR, CIC 19nn, p. xx, 19nn désignant l'année de la CIC.
} 
l'Instruction publique, qui présidait cette dernière séance, remit la médaille d'or de la SCI au savant et prix Nobel toulousain. Il a conclu en insistant sur les rapports étroits unissant la science et l'industrie. L'année suivante, Rivals obtenait la création d'un Laboratoire national des matières grasses associé à son Institut ${ }^{33}$.

Revenons sur les liens évidents pour les congressistes de l'Union internationale de chimie avec la SCI. Un rappel des circonstances de la création de cette union est ici nécessaire.

À la fin de la guerre, l'Association internationale des académies scientifiques décidait de dissoudre les anciennes associations internationales pour en créer des nouvelles d'où les puissances centrales seraient exclues. En chimie, il s'agissait de dissoudre la récente Association internationale des sociétés chimiques (AISC, fondée en 1911 par Haller, Wilhelm Ostwald et William Ramsay), puis de créer ce qui deviendrait en 1919 l'Union internationale de chimie pure et appliquée (UICPA) ${ }^{34}$. Les deux sociétés de chimie industrielles, britannique et française, apportèrent un soutien total à cette création, tant logistique (accueil dans des lieux réservés par elles, alternativement à Londres et à Paris), financière (tous les frais furent supportés par ces deux associations en 1918 et 1919), qu'administrative (des membres des deux sociétés ont contribué à la réalisation des statuts, au règlement et à la charge

\footnotetext{
${ }^{33}$ Voir Tachoire Henri, « une brève histoire de la Faculté des sciences de Marseille » : http://sites.univ-provence.fr/wabim/d_sc1922/\#breve. Extrait d'une brochure publiée par l'université de Provence, Service des publications, 1996.

${ }^{34}$ Le français est alors la langue officielle de l'Union. En 1930 elle prend le nom d'Union internationale de chimie (UIC), pour redevenir UICPA après la Seconde guerre mondiale. À la fin des années 1960, l'anglais devient langue officielle, et le nom International Union of Pure and Applied Chemistry donne IUPAC.
} 
administrative $)^{35}$. P. Kestner et J. Gérard se sont pleinement investis. Le premier a usé de sa notoriété pour la prise de contacts, et contribué ensuite à l'activité de certaines commissions dont celle des brevets. Le second - il avait alors 28 ans - a été élu secrétaire général de 1'UICPA. Il devait le rester jusqu'en 1944. Il était déjà secrétaire général de la SCI, et secrétaire général de la Fédération nationale des associations de chimie (FNAC), créée quelques semaines plus tôt pour représenter la France au sein de l'Union. Cette FNAC, comme l'UICPA, avait son siège social dans le même bâtiment que la SCI. Gérard était également engagé dans plusieurs autres instances tant internationales que commerciales. Doté d'un sens aigu de l'organisation, et d'une puissance de travail hors du commun, il se faisait le chantre d'une organisation rationnelle de tous les secteurs du monde industriel s'inspirant pour cela des doctrines américaines du taylorisme, où la science devait être impliquée autant que d'être appliquée.

Gérard se met rarement en avant, mais est présent et actif partout, sait considérer tous les aspects d'un problème et y répondre ; il se rend indispensable, tire toutes les ficelles, et possède un carnet d'adresses exceptionnel. Il est considéré comme l'éminence grise, la « cheville ouvrière ». Si le président est l'homme du devant de la scène, le secrétaire général, dans les coulisses, tient le pouvoir effectif. Rapidement, Gérard devient un homme incontournable. C'est donc sous son impulsion, discrète mais permanente, que les liens entre les conférences internationales de chimie et les congrès de chimie industrielle sont particulièrement forts jusqu'en 1928. Après cette date,

\footnotetext{
${ }^{35}$ Fauque D., «French Chemists and the International Reorganisation of Chemistry after World War I », Ambix, 58/2, 116-135 (July 2011).
} 
c'est moins évident. Des tensions apparaissent à l'Union qui réforme ses statuts et règlement; les liens avec la SCI britannique ne sont plus tout à fait ceux d'après la guerre; Kestner se retire de la vie publique. La SCI britannique prend du recul ${ }^{36}$.

En lisant les comptes rendus des deux types de manifestations, il apparaît que dans le cadre du CCI, des vœux émis par les CIC trouvent un début de réalisation, ou sont discutés dans ce but, de même des vœux émis par des groupes des CCI sont portés sur la scène internationale par le biais des CIC. Le va-et-vient est subtil entre les deux organismes, et mériterait une attention spécifique. L'étude des compositions des délégations présentes au cours des CCI et des CIC permettrait de dégager de façon précise les liens effectifs entre les deux mondes. L'un comme l'autre sont des manifestations généralistes. Peu à peu des associations internationales spécialisées se créeront, relativisant du même coup l'impact législatif notamment sur les standards que pouvaient avoir des organismes généralistes.

D'après le règlement de l'Union, un comité consultatif devait assurer la représentation complète de la chimie appliquée auprès de l'Union pour l'étude de toutes les questions d'ordre chimique. Au début des conférences de chimie, des questions d'ordre technique et industriel ont été mises à l'étude : brevets, tout ce qui touche aux combustibles, produits céramiques, produits industriels et technologiques de référence... Mais le Comité consultatif n'a pas réellement fonctionné, et l'article le concernant fut supprimé dans les

\footnotetext{
${ }^{36}$ Voir Fauque D., «French Chemists », op. cit., note 35. Voir Mackie Robin et Roberts Gerrylynn K., «Un secteur à part ? Les chimistes industriels et la Society of Chemical Industry dans le contexte de la communauté chimique britannique », in Chimie et industrie en Europe, Fell U. dir., op. cit., note 1, p. 127-147 (trad. Nathalie Jas).
} 
nouveaux statuts de l'Union en 1928. L'ordre des spécialités représentées dans ce comité correspondait à peu de choses près à celui de la CDU.

\section{Travail des sections}

Les congrès de 1921 et 1922 sont en quelque sorte à la recherche d'une structure définitive. Celle-ci est mise en place en 1923 ; les congrès comportent maintenant six groupes, chacun étant divisé en sections. Les groupes sont les suivants (tableau 4) : Groupe I, Usine et laboratoire; Groupe II, Combustibles; Groupe III, Métallurgie et industries minérales; Groupe IV, Industries organiques ; Groupe V, Agronomie et industries agricoles ; Groupe VI, Organisation économique. Chaque section peut se réunir une demijournée, voire une journée entière.

$\mathrm{Au}$ cours des congrès, des journées à thème s'établissent successivement pour les combustibles, pour le caoutchouc, pour l'agriculture, ou pour la métallurgie. Aux conférences générales d'ouverture ou de clôture vont s'ajouter peu à peu des conférences de groupes offrant une synthèse sur une branche particulière, souvent liée d'ailleurs aux événements extérieurs, ou aux visites d'usines projetées. Donnons quelques exemples. Des conférences sur la chimie et la couleur, la chimie et la céramique, la chimie des parfums, les arts décoratifs et les matières plastiques en 1925, préparent la visite de l'Exposition des arts décoratifs. En 1926, dans le Groupe III, la métallurgie au Katanga, ou la « vie intime » du verre, en 1927 ; dans le groupe VI, une conférence sur la chimie et la presse aide à la visite du journal L'Intransigeant. À Strasbourg (1928), deux journées de 
spécialité sont organisées, avec une conférence chacune (Journée des combustibles, Journée de la métallurgie et des industries minérales). En 1931, à Paris, pendant le congrès de chimie industrielle, un congrès sur les applications industrielles du caoutchouc, organisé également par la SCI se tient en relation avec l'Exposition coloniale. Mais les journées de spécialité peuvent être suscitées par une question posée lors d'un congrès précédent. C'est le cas pour le congrès sur les combustibles liquides d'octobre 1922, décidé après le CCI de Marseille (juillet 1922).

\section{À l'intérieur des sections}

Le congrès a pour objectif d'informer la communauté sur les innovations et les moyens de rendre plus rationnelle l'organisation de tous les aspects de l'industrie ; il est aussi l'occasion de discussions dans le but d'aboutir à des règles communes de production ou de commerce. La SCI, militante du taylorisme et de l'organisation technologique, économique et documentaire la plus poussée, veut aussi apporter des résultats d'enquête économique sur l'état industriel de telle ou telle branche ou de tel ou tel pays. Au fil des pages des comptes rendus des congrès, les sujets les plus récurrents portent sur la standardisation et la demande de législation, le développement de l'analyse physicochimique, les combustibles et carburants, la question de l'azote et des engrais, les tanneries, le caoutchouc, "l'antioxydation", les brevets et la documentation. Nous allons préciser ciaprès des aspects de ces différents items.

Les questions de standardisation apparaissent surtout en chimie analytique, où l'on demande expressément des réactifs et des échantillons standards, puis des méthodes standards. La question de 
l'établissement d'étalons chimiques a été débattue dès le premier CCI. En 1921, la France décide d'établir un codex des réactifs analytiques, décision faisant suite à celle prise par la Commission des produits purs pour recherche de la CIC de Bruxelles $(1921)^{37}$. Présenté par André Kling, le codex français est adopté au CCI de 1922, puis reconnu provisoirement par la CIC de Cambridge (1923) sous le nom de codex des réactifs «type congrès 1922 », enfin adopté définitivement à la CIC de Copenhague (1924) ${ }^{38}$. Kling, président de la commission de chimie analytique succédant à la commission des produits purs pour recherche en 1927, joue un rôle majeur dans la réalisation des codex de réactifs toutes catégories jusqu'en 1928 où son rapport général est adopté par la CIC de la Haye. Il comptait beaucoup sur le congrès international de chimie prévu en Espagne en 1932 pour faire avancer la question de la standardisation des réactifs, mais les événements en décideront autrement. En 1936, à Lucerne, une commission des réactifs nouveaux succède à la commission de chimie analytique présidée par Cornelis J. van Nieuwenburg, professeur de chimie analytique à l'université technique de Delft, mais elle ne se remettra au travail qu'après la Libération, une fois renouvelée. Le sujet ne fut plus abordé sous cette forme aux CCI des années 1930. Cet exemple de la réalisation de codex des réactifs analytiques est typique des liens qui s'établissent entre les deux types de manifestations, les CCI et les CIC jusqu'à la fin des années 1920 .

\footnotetext{
${ }^{37}$ CR, CIC 1921, p. 14 et p. 53.

${ }^{38}$ Le codex est publié dans Ch\&I, 9/1 (janvier 1923). Deschiens M., « Troisième congrès de chimie industrielle », Ch\&I, 11/NS, 7 (mai 1924). Voir CR, CIC 1923, p. 53-54 et les rapports p. 103-109. Voir CR, CIC 1924, p. 47 et Kling A., « Rapport général », p. 66-90.
} 
En 1925, on souhaite qu'un cahier des charges sur la verrerie jaugée et graduée (forme, contenance, graduations, précision) soit établi et serve de référence. On demande également une méthode d'analyse des boissons alcoolisées qui soit "universelle", vœu que la SCI transmettra au ministère des Affaires étrangères.

Le groupe des combustibles et carburants travaille sur un sujet de plus en plus sensible, en particulier sur la recherche d'un « carburant national », la recherche d'un carburant de synthèse et les essais de différents carburants. C'est l'objet d'un congrès spécifique en octobre 1922, organisé par la SCI, et de plusieurs journées spécialisées au cours des congrès suivants ${ }^{39}$. La succession des interventions sur la période 1921-1938 permet de saisir l'accélération et la mutation des méthodes de production industrielle des combustibles (notamment sur la pyrogénation, le craquage et les catalyseurs, pour l'obtention d'hydrocarbures légers). Tandis que la question n'est plus abordée aux CIC à partir de 1930.

La question de l'azote est très présente dans les premières années de la décennie 1920. Elle s’estompe à partir des années 1930, sauf en ce qui concerne les engrais. La référence à l'Allemagne notamment dans des articles de synthèse publiés dans Chimie \& Industrie, les conférences et publications de Georges Claude et de Camille Matignon sur le sujet, montrent l'importance que ce secteur a

\footnotetext{
${ }^{39}$ Voir Molles Camille, « III - La Société de Chimie industrielle face au problème de l'alcool», L'Actualité chimique, 382-383, 113-117 (février-mars 2014), (Dossier: Les chimistes, leurs institutions et leurs sociétés savantes entre les deux guerres).
} 
joué dans l'image de l'industrie chimique française après la Grande guerre $^{40}$.

Comme il a été dit précédemment, l'industrie de la tannerie est un cas intéressant à suivre. Elle semble être la première à faire état de l'utilisation de la notion de $\mathrm{pH}^{41}$. L'industrie de la tannerie est suivie par l'industrie des matières alimentaires et les conserveries qui utilisent de plus en plus le pH pour suivre l'évolution des réactions chimiques dans leur processus de production. Le $\mathrm{pH}$ est aussi utilisé par l'industrie du papier qui en fait état en $1927^{42}$. Au cours des CCI, une codification des analyses des matières tannantes est établie, si bien qu'un accord international semble obtenu en 1938. En effet, en 1936, un long rapport sur les méthodes officielles d'analyse, présenté par F. Pothier, de la section française de la SICIC, est soumis à l'examen de l'Union internationale de chimie. En 1938, à la CIC de Rome, ces méthodes sont adoptées après consultation des deux organismes internationaux concernés, la SICIC et l'IVLIC ${ }^{43}$.

\footnotetext{
${ }^{40}$ Voir Emptoz G., « II - La nouvelle chimie de l'azote », op.cit. note 18 ; Baillot Rémi, George Claude, le génie fourvoyé, créateur d'Air liquide, du tube à néon, de l'énergie thermique des mers..., EDP Sciences, Les Ulis, 2010.

${ }^{41}$ Voir note 27. En 1924, la section française charge la SCI d'être le relais après des diverses administrations dans le but d'uniformiser les méthodes étudiées et adoptées par cette section. Voir "Quatrième congrès de chimie industrielle », Ch\&I, NS, 23 (septembre 1925).

42 « Sixième congrès de chimie industrielle », Ch\&I, NS, 37 (mai 1927). « Septième congrès de chimie industrielle », Ch\&I, NS, 28 (avril 1928).

${ }^{43}$ Pothier F., « Méthodes officielles d'analyse des produits de l'industrie du cuir, rapport», in $C R, C I C$ 1936, p. 49-63. Meunier L., "Commission chargée d'examiner le rapport sur les méthodes officielles d'analyse des produits de 1'industrie du cuir » in CR, CIC 1938, p. 53. SICIC: Société internationale des chimistes de l'industrie du cuir. IVLIC : Internationaler Verein der Leder Industrie Chemiker.
} 
À contrario, l'industrie du caoutchouc participe tardivement en tant que telle, mais la question est discutée à chaque congrès, qui finit par déboucher sur une manifestation spécifique en $1931^{44}$.

L'anti-corrosion est un domaine qui va surtout être abordé à partir des années 1930. Suite à leurs travaux sur la stabilisation de l'acroléine pendant la guerre, Charles Moureu et Charles Dufraisse découvre le phénomène d'anti-oxydation. Moureu donne une conférence générale sur le sujet en $1926^{45}$. Cela conduit à l'invention d'alliages aux propriétés particulières et d'aciers inoxydables. Le sujet est développé à la fin des années 1930, entre autres par Charles Dufraisse, et particulièrement au cours d'une journée «anticorrosion » organisée à la Maison de la Chimie en $1938^{46}$.

Dès la fondation de l'Union internationale de chimie, une commission du brevet industriel est créée dont Kestner fut le rapporteur, afin de débattre sur les moyens à entreprendre pour aboutir à des mesures codifiant la propriété industrielle et donc la réglementation d'un brevet international. Il y a eu beaucoup d'espoirs mis dans l'Union internationale pour cette question dont la demande d'une conférence internationale sur le sujet ${ }^{47}$. Aux conférences de 1922 et de 1924, Kestner fait le point, soulignant la difficulté d'arriver

\footnotetext{
44 « Congrès international pour le développement des applications du caoutchouc », Paris, 29 septembre- $1^{\mathrm{er}}$ octobre 1931, Ch\&I, NS (1932). 18 communications.

${ }^{45}$ Moureu Ch., « La catalyse antioxygène », in " Sixième congrès », op. cit. note 42, p. 101-109.

${ }^{46}$ « Journées de la lutte contre la corrosion », Paris, 21-23 novembre 1938, Ch\&I, NS (1938). Elles se tiennent à la Maison de la Chimie, et sont organisées par la SCI et le Centre de perfectionnement technique de la Maison de la Chimie.

${ }^{47}$ CR, CIC 1920, p. 49. « Brevet international», CR, CIC 1921, p. 50. E. Paterno, « Rapport présenté au nom du Consiglio nazionale di chimica », CR, CIC 1921, p. 111-112.
} 
à un consensus ${ }^{48}$. Le conseil économique de la SDN s'en préoccupe également ${ }^{49}$. Au congrès de chimie industrielle, la question est très débattue à partir de 1925, avec le projet de loi déposé par le gouvernement le 29 juillet 1924, et dont les rapports viennent d'être rendus. C'est donc là encore un champ d'échanges entre les CIC et les $\mathrm{CCI}^{50}$. Mais à nouveau, la question s'estompe au bout de quelques congrès, comme aux CIC, à partir de $1930^{51}$.

Quant à la documentation, la demande d'accès rapide à toute information est grande, sujet sur lequel s'efforce de répondre la SCI. La discussion de cette question suivra les évolutions de la fondation de l'Office international de chimie (1927) et l'installation de la Maison de la Chimie (1934). En 1926, une part importante sera dédiée à ce sujet ${ }^{52}$. Elle est à mettre en parallèle avec la préparation de la conférence internationale pour la création de l'Office international de chimie qui se tiendra au lendemain de la célébration du centenaire de Marcellin Berthelot en 1927, pour le lancement de la création de la Maison de la Chimie ${ }^{53}$.

\footnotetext{
${ }^{48}$ Kestner P., « Rapport présenté au nom de la Fédération des associations de chimie de France », CR, CIC 1922, p. 125-131. CR, CIC 1924, p. 168-175.

${ }^{49}$ Voir à ce sujet Galvez-Béhar Gabriel, «The "French connection" : French Scientists and International Debates on Scientific Property during the Interwar Period », (2013) : https://halshs.archives-ouvertes.fr/halshs-00839580

${ }^{50}$ Monteilhet Antonin, «Modifications à apporter au projet de loi sur les brevets actuellement en discussion devant le Sénat », in «Septième Congrès », op. cit., note 42, p. 950-952, voir p. 950.

${ }^{51}$ Voir les mesures prises sur le plan international par des organismes spécifiques indépendants de l'union.

52 « Groupe VI, Organisation économique », in « Sixième congrès », op. cit., note 42, p. 42. Texte des communications, p. 727-737.

${ }^{53}$ Fauque D., « La documentation », op. cit., note 30.
} 
Des questions ne seront pas abordées au cours des congrès, comme la nomenclature qui relève spécifiquement de l'Union. Mais aux CCI, des vœux seront énoncés sur ce sujet, notamment il est demandé une nomenclature précise pour les différents combustibles au début des années 1920. En 1925, on souhaite que 1'UICPA se saisisse de la question de la terminologie des résines artificielles, et l'inscrive à l'ordre du jour d'une prochaine $\mathrm{CIC}^{54}$.

\section{Les voux}

Ainsi, les résultats des discussions prennent souvent la forme de vœux dont la présence reste constante au cours des vingt années étudiées. Au début des années 1920, outre ce que nous avons déjà dit, les questions suivantes sont les plus discutées : pouvoir calorifique des combustibles, et du gaz d'éclairage en particulier; protection des minerais radioactifs de Madagascar (interdiction de vente à des étrangers) ; législation sur les savons dont l'imposition d'une étiquette indiquant la composition; législation sur les matières alimentaires, dont les moyens physicochimiques de conservation ; législation sur les matières grasses (définition, composition, pourcentage), en particulier sur les huiles d'animaux marins, et cela pendant plusieurs années. En 1929, une demande de législation sur les vitamines ajoutées aux aliments industriels est posée. Une discussion s'établit sur une législation en cours d'étude : la loi sur l'exercice de la pharmacie, et le statut des médicaments de synthèse ${ }^{55}$.

\footnotetext{
54 «Cinquième congrès de chimie industrielle », Ch\&I, 16/3bis, 26 (septembre 1926).

55 « Deuxième congrès », op. cit., note 31 , p. 471-472; «Troisième congrès ", op. cit., note 38, vœu de la section 8, p. 7-8.
} 
Des voix s'élèvent demandant que la protection de l'industrie française des colorants soit renforcée, une révision des tarifs douaniers sur le charbon, une limitation des importations à prix de revient plus bas pour favoriser l'industrie nationale... Dans la crise économique qui sévit et la dévaluation du mark qui pénalise le franc fort, les chimistes académiques comme A. Béhal, A. Haller ou Ch. Moureu, n'hésitent pas à lancer un appel patriotique dans la Revue scientifique $(1922)^{56}$. En 1925, un vœu est émis pour demander aux pouvoirs publics de ne pas sacrifier la prospérité et l'avenir de la chimie française et de cette belle industrie des colorants «quand viendra l'examen de la question douanière $»^{57}$. En même temps, le monde académique comme le monde industriel demandent une réforme de la formation du chimiste ${ }^{58}$.

En octobre 1929, quelques jours avant le krach de Wall Street, on envisage les conditions de réalisation d'une Entente économique européenne au moment où des négociations diplomatiques et économiques s'ouvrent ${ }^{59}$. Cette situation traduit bien le climat paneuropéen des années 1928-1929, dans le sillage du discours d'Aristide Briand à l'assemblée de la Société des nations le 5 septembre 1929. Le Français suggérait la formation d'une « union» ou d'une «fédération européenne» ainsi que le développait le

\footnotetext{
${ }^{56}$ Op. cit., note 28.

57 « Cinquième congrès », op. cit., note 54, p. 154 ; Guiselin A., « La réforme douanière », in « Cinquième congrès », op. cit. note 54, p. 721-724.

${ }^{58}$ Sisley Paul, « La chimie et la couleur », in « Cinquième congrès », op. cit. note 54, p. 147-154, voir p. 154. Sordes René, «L'enseignement de la chimie », in « Cinquième congrès », op. cit., note 54, p. 704-708.

59 « Neuvième congrès de chimie industrielle », Ch\&I, NS, 19 et 290 (mars 1930). Le congrès se déroule à Barcelone du 13 au 19 octobre; le krach a lieu le jeudi 24 octobre.
} 
diplomate polyglotte Richard Nikolaus von Coudenhove-Kalergi trois ans auparavant: un plan "paneuropéen » pouvait seul établir une sécurité en Europe ; la Grande crise allait balayer cela ${ }^{60}$.

La SCI joue donc le rôle de relais. Les demandes qu'elle prend en charge sont soumises aux autorités compétentes : administrations, ministères, ou organismes spécialisés, mais aussi au Bureau international du travail, et aux organismes internationaux (UICPA, en particulier).

Outre les vœux, les congressistes rédigent des questions à mettre à l'ordre du jour du congrès suivant : « Les congrès précédents annoncent en effet les questions à l'ordre du jour, les congrès qui suivent voient des réalisations pratiques, des mises au point, l'ébauche de théories ou de pratiques nouvelles $»^{61}$. C'est ainsi qu'a été décidée la tenue de conférences spécifiques comme celles des combustibles (1922), du caoutchouc (1931), de la corrosion (novembre 1938), les matières plastiques (juin 1939), ou de la documentation. Des textes ou motions sont également votés au cours du congrès.

\section{Conclusion}

Des comptes rendus sont publiés en volumes séparés à partir du troisième congrès (1923), en numéros spéciaux de la revue Chimie \& Industrie, quelques mois après l'événement, en général entre février et mai de l'année suivante. Une grande majorité des communications

\footnotetext{
${ }^{60}$ Renouvin Pierre (dir.), Histoire des relations internationales, t. VII. Les crises $d u$ $X X^{e}$ siècle, I. de 1914 à 1929, Hachette, Paris, 1969, p. 343. Guieu Jean-Michel, Gagner la paix, 1914-1929, Éditions du Seuil, Paris, 2015, p. 465-466.

${ }^{61}$ « Sixième congrès », op. cit., note 42, p. 26.
} 
y sont présentes. D'autres sont publiées dans le périodique, parfois avant le congrès, dans le cas d'une conférence notamment. Relire le périodique à la lumière de ces observations permettrait de mettre en parallèle les thèmes dominants du congrès et les thèmes développés dans le périodique. Quelques articles généraux semblent publiés deux fois, soit sous forme d'extrait, soit travaillé différemment, dans le numéro spécial et dans le périodique.

$\mathrm{Au}$ final, on peut considérer que la SCI, dont la devise est « militer et servir », veut tout faire pour répandre la bonne parole de l'organisation rationnelle de l'usine comme du laboratoire et pour réaliser ce que son programme initial annonçait dans la plaquette de décembre 1917. Elle a à sa disposition deux organes, la revue et le congrès où des milliers de gens se croisent, des centaines se parlent et échangent, et qui est, en fait, sans que son titre ne le laisse paraître, un véritable congrès international. Il y a encore beaucoup à apprendre de la lecture de ces textes. Étudier une spécialité permettrait de suivre l'évolution de l'introduction des méthodes modernes dans son domaine de production (comme l'usage du $\mathrm{pH}$, la micrographie $\mathrm{X}$, la spectroscopie, etc.).

Il faudrait aussi s'intéresser aux acteurs, dont certains sont présents à la fois dans les congrès et les conférences internationales de chimie. Notons le nom de Camille Matignon, rédacteur en chef de la revue et président de la commission de la documentation à l'Union, ou celui de Kling, directeur du laboratoire municipal, et président de la commission de chimie analytique de l'Union. Notons des étrangers, membres de l'UICPA à un titre ou un autre, souvent présents et intervenants dans les congrès: Frédéric Swarts (Belgique), un des 
premiers membres de l'UICPA à qui la SCI décernera la grande médaille d'or (1926), à Einar Biilmann, devenant membre d'honneur de la SCI (1931), alors qu'il est président de l'Union, avec la grande médaille d'or à Emil Votocek (Prague, 1932), au Russe Vladimir Ipatieff (1928), dans le même temps que l'URSS est invitée aux conférences successives de l’Union (1927, 1928 et 1930).

Le soutien public des hommes politiques, non seulement moral mais aussi par leur présence, est incontestable. Nul doute que ces congrès ont favorisé la modernisation de l'industrie chimique française, et l'établissement effectif d'un certain nombre de normes industrielles et de lois relatives au commerce mais aussi aux questions de santé publique. La décennie 1920 apparaît comme une période de grands bouleversements dans ce domaine. S'informer pour s'adapter était devenu crucial pour beaucoup, quelque soit la branche considérée.

Après la crise de 1929, il apparaît une sorte de retour à l'entre soi. Les communications gagnent en technicité et en savoir savant. Les liens avec l'Union sont distendus. Les organisateurs et les rapporteurs notent que l'organisation rationnelle du travail scientifique, technique ou industriel, s'est largement répandue, et surtout que le laboratoire est de plus en plus présent dans l'usine. L'avenir est vraiment ouvert aux ingénieurs et aux techniciens. 


\section{Annexe}

Tableau 1. Tableau chronologique des congrès de chimie industrielle (CCI), 19211939.

\begin{tabular}{|c|c|c|c|}
\hline Année & $\begin{array}{l}\text { Période CCI } \\
1921-1939\end{array}$ & $\begin{array}{l}\text { Choix d'événements } \\
\text { contemporains }\end{array}$ & $\begin{array}{l}\text { IUPAC - } \\
\text { Conférences }\end{array}$ \\
\hline 1920 & & & $\begin{array}{l}\text { Rome } \\
(22-24 / 6)\end{array}$ \\
\hline 1921 & $10-13 / 10-$ Paris & $\begin{array}{l}\text { Exposition de chimie (Paris, 7-16 } \\
\text { octobre) }\end{array}$ & $\begin{array}{l}\text { Bruxelles } \\
(27-30 / 6)\end{array}$ \\
\hline 1922 & $\begin{array}{l}2-8 / 7- \\
\text { Marseille }\end{array}$ & $\begin{array}{l}\text { Exposition coloniale (Marseille, } \\
\text { avril-novembre) } \\
\text { Congrès international des } \\
\text { combustibles liquides (Paris, 9- } \\
15 \text { octobre - esplanade des } \\
\text { Invalides) } \\
\text { Exposition sur les combustibles } \\
\text { liquides (Paris) }\end{array}$ & $\begin{array}{l}\text { Lyon } \\
\left(27 / 6-1^{\mathrm{er}} / 7\right)\end{array}$ \\
\hline 1923 & 21-26/10-Paris & $\begin{array}{l}\text { Centenaire de la naissance de } \\
\text { Pasteur (mai) }\end{array}$ & $\begin{array}{l}\text { Cambridge } \\
(17-20 / 6)\end{array}$ \\
\hline 1924 & $\begin{array}{l}15-21 / 6- \\
\text { Bordeaux }\end{array}$ & Foire (Bordeaux, 15-30 juin) & $\begin{array}{l}\text { Copenhague } \\
\left(26 / 6-1^{\mathrm{er}} / 7\right)\end{array}$ \\
\hline 1925 & 4-11/10 - Paris & $\begin{array}{l}\text { Exposition des arts décoratifs et } \\
\text { industriels modernes (Paris) } \\
\text { Exposition sur la chimie et les } \\
\text { arts décoratifs (Paris, } 28 \text { avril-25 } \\
\text { octobre) }\end{array}$ & $\begin{array}{l}\text { Bucarest } \\
(22-25 / 6)\end{array}$ \\
\hline 1926 & $\begin{array}{l}\text { 25/9-2/10- } \\
\text { Bruxelles }\end{array}$ & & $\begin{array}{l}\text { Washington } \\
(13-15 / 9)\end{array}$ \\
\hline 1927 & 16-22/10 - Paris & $\begin{array}{l}\text { Centenaire M. Berthelot (23-26 } \\
\text { octobre) } \\
\text { Conférence internationale pour la } \\
\text { création de l'office international } \\
\text { de documentation (Paris, } 27 \\
\text { octobre) }\end{array}$ & $\begin{array}{l}\text { Varsovie } \\
(4-14 / 9)\end{array}$ \\
\hline 1928 & $\begin{array}{l}23-29 / 7- \\
\text { Strasbourg }\end{array}$ & $\begin{array}{l}\text { Centenaire de P. Schützenberger } \\
\text { à Strasbourg } \\
\text { Excursions et visites d'usines à } \\
\text { Luxembourg ( } 29 \text { juillet) }\end{array}$ & $\begin{array}{l}\text { La Haye } \\
(18-24 / 7)\end{array}$ \\
\hline 1929 & $\begin{array}{l}13-19 / 10- \\
\text { Barcelone }\end{array}$ & $\begin{array}{l}\text { Exposition internationale de } \\
\text { Barcelone (20 mai 1929-15 } \\
\text { janvier 1930) }\end{array}$ & \\
\hline 1930 & 7-13/9 - Liège & $\begin{array}{l}\text { Centenaire de l'Indépendance. } \\
\text { Exposition internationale de la } \\
\text { grande industrie, science et } \\
\text { application et d'art wallon }\end{array}$ & $\begin{array}{l}\text { Liège } \\
(14-20 / 9)\end{array}$ \\
\hline 1931 & $\begin{array}{l}27 / 9-1^{\mathrm{er}} / 10- \\
\text { Paris }\end{array}$ & $\begin{array}{l}\text { Exposition coloniale (Paris- } \\
\text { Vincennes, } 6 \text { mai-15 novembre) }\end{array}$ & \\
\hline
\end{tabular}




\begin{tabular}{|c|c|c|c|}
\hline & & $\begin{array}{l}\text { Congrès international pour le } \\
\text { développement des applications } \\
\text { du caoutchouc (Paris, } 29 \\
\text { septembre- } 1^{\text {er }} \text { octobre) }\end{array}$ & \\
\hline 1932 & $\begin{array}{l}25 / 9-1^{\mathrm{er}} / 10- \\
\text { Prague }\end{array}$ & & $\begin{array}{l}\text { Madrid (annulé et } \\
\text { reconduit à 1934) }\end{array}$ \\
\hline 1933 & 24/9-30/9 - Lille & $\begin{array}{l}\text { Suivi d'un hommage à Jean } \\
\text { Effront à Bruxelles ( } 1^{\text {er }} \text { octobre) }\end{array}$ & \\
\hline 1934 & 22-27/10 - Paris & $\begin{array}{l}\text { Exposition de la chimie, section } \\
\text { de } 1 \text { 'outillage de laboratoire } \\
\text { (Paris, } 27 \text { octobre- } 1^{\text {er }} \text { novembre) } \\
\text { Symposium sur la normalisation } \\
\text { de la verrerie de laboratoire } \\
\text { (Paris, } 28 \text { octobre) } \\
\text { Inauguration de la Maison de la } \\
\text { Chimie ( } 2 \text { décembre) }{ }^{62}\end{array}$ & $\begin{array}{l}\text { Madrid } \\
(5-11 / 4) \\
\text { (joint au IX } \\
\text { Congrès } \\
\text { international de } \\
\text { chimie) }\end{array}$ \\
\hline 1935 & $\begin{array}{l}23-28 / 9- \\
\text { Bruxelles }\end{array}$ & $\begin{array}{l}\text { Exposition universelle et } \\
\text { internationale (Bruxelles, mai- } \\
\text { novembre) }\end{array}$ & \\
\hline 1936 & Espagne & $\begin{array}{l}\text { Annulé pour cause de guerre } \\
\text { civile }\end{array}$ & $\begin{array}{l}\text { Lucerne et Zurich } \\
(12-22 / 8)\end{array}$ \\
\hline 1937 & $\begin{array}{l}26 / 9-3 / 10- \\
\text { Paris }\end{array}$ & $\begin{array}{l}\text { Célébration des vingt ans de la } \\
\text { SCI } \\
\text { Exposition sur l'outillage de } \\
\text { laboratoire }\end{array}$ & \\
\hline 1938 & $\begin{array}{l}\text { 22/9-2/10- } \\
\text { Nancy }\end{array}$ & $\begin{array}{l}\text { Écourté le } 24 \text { pour cause de } \\
\text { mobilisation } \\
\text { Journée des engrais (Paris, 12-16 } \\
\text { novembre) } \\
\text { Journée contre la corrosion } \\
\text { (Paris, 21-23 novembre) }\end{array}$ & $\begin{array}{l}\text { Rome } \\
(15-21 / 5) \\
\text { (joint au } X^{\mathrm{e}} \\
\text { Congrès } \\
\text { international de } \\
\text { chimie) }\end{array}$ \\
\hline 1939 & $\begin{array}{l}24 / 9-2 / 10- \\
\text { Varsovie }\end{array}$ & $\begin{array}{l}\text { Annulé pour cause de guerre } \\
\text { Journée des matières plastiques } \\
\text { (Paris, } 10-18 \text { juin) }\end{array}$ & \\
\hline Année & $\begin{array}{l}\text { Période CCI } \\
\text { 1921-1939 }\end{array}$ & $\begin{array}{l}\text { Choix d'événements } \\
\text { contemporains }\end{array}$ & $\begin{array}{l}\text { IUPAC - } \\
\text { Conférences }\end{array}$ \\
\hline
\end{tabular}

\footnotetext{
${ }^{62}$ Fauque D., « La documentation», RHS, op. cit., note 30, p. 65.
} 
Tableau 2. Congrès de chimie industrielle (CCI). Composition des six groupes à partir de 1923

\begin{tabular}{|c|c|c|}
\hline Groupe & $\begin{array}{l}\text { Titre } \\
(1923)\end{array}$ & $\begin{array}{l}\text { Titre complet (légères évolutions au cours des } \\
\text { congrès) ou titres des sections }\end{array}$ \\
\hline $\begin{array}{l}\text { Groupe } \\
\text { I }\end{array}$ & $\begin{array}{l}\text { Usine } \\
\text { laboratoire }\end{array}$ & $\begin{array}{l}\text { Chimie analytique, outillage du laboratoire, outillage } \\
\text { de l'usine, industries frigorifiques, eaux }\end{array}$ \\
\hline $\begin{array}{l}\text { Groupe } \\
\text { II }\end{array}$ & Combustibles & $\begin{array}{l}\text { Combustibles solides, liquides et gazeux, chauffage, } \\
\text { éclairage, graissage. }\end{array}$ \\
\hline $\begin{array}{l}\text { Groupe } \\
\text { III }\end{array}$ & $\begin{array}{l}\text { Métallurgie } \\
\text { industries } \\
\text { minérales }\end{array}$ & $\begin{array}{l}\text { Métallurgie, électrométallurgie, métaux précieux. } \\
\text { Grande industrie chimique, électrochimie, petite } \\
\text { industrie chimique, terres rares, corps radioactifs. } \\
\text { Chaux, ciments, matériaux de construction, verrerie, } \\
\text { céramique, émaillerie. }\end{array}$ \\
\hline $\begin{array}{l}\text { Groupe } \\
\text { IV }\end{array}$ & $\begin{array}{l}\text { Industries } \\
\text { organiques }\end{array}$ & $\begin{array}{l}\text { Matières colorantes, textiles naturels, teinture, } \\
\text { impression, blanchiment et apprêts, poudres et } \\
\text { explosifs. } \\
\text { Produits pharmaceutiques, produits photographiques, } \\
\text { essences, parfums naturels et synthétiques. } \\
\text { Matières grasses, savons, bougies, glycérines. } \\
\text { Caoutchouc et succédanés, résines, couleurs laques, } \\
\text { vernis, cires et produits d'entretien. } \\
\text { Cellulose, papier, matières plastiques, textiles } \\
\text { artificiels. } \\
\text { Extraits tinctoriaux et tannants, industries de la } \\
\text { tannerie et annexes. }\end{array}$ \\
\hline $\begin{array}{l}\text { Groupe } \\
\text { V }\end{array}$ & $\begin{array}{l}\text { Agronomie } \\
\text { industries } \\
\text { agricoles }\end{array}$ & $\begin{array}{l}\text { Industries de la fermentation, œnologie, cidrerie, } \\
\text { brasserie, distillerie, sucrerie, féculerie, amidonnerie, } \\
\text { glucoserie. } \\
\text { Laiterie, matières alimentaires, sols, utilisation des } \\
\text { engrais, agriculture }\end{array}$ \\
\hline $\begin{array}{l}\text { Groupe } \\
\text { VI }\end{array}$ & $\begin{array}{l}\text { Organisation } \\
\text { économique }\end{array}$ & $\begin{array}{l}\text { Enseignement, organisation économique, } \\
\text { organisation, sources et débouchés, sociologie, } \\
\text { hygiène industrielle, législation. }\end{array}$ \\
\hline
\end{tabular}


